\title{
Über Wachstumsterme in den Bilanzgleichungen der Kontinuumsmechanik, speziell beim Wachstum von biologischen Materialien
}

\author{
Von \\ Hans Irschik \\ (Vorgelegt in der Sitzung der math.-nat. Klasse am 14. Oktober 2004 \\ durch das w. M. Franz Ziegler)
}

\section{Zusammenfassung}

Wachstumsterme finden sich in letzter Zeit immer häufiger in Theorien zur Beschreibung des Wachstums biologischer Materialien, z. B. bei mechanischen Theorien zum Tumorwachstum. In der vorliegenden Arbeit werden zunächst Beziehungen hergeleitet, welche notwendigerweise gelten müssen zwischen den Wachstumstermen in den fundamentalen Bilanzgleichungen der Kontinuumsmechanik, also für Masse, Impuls, Drall und Energie, und den Wachstumstermen in den abgeleiteten Bilanzgleichungen, also für kinetische Energie, Impulsmoment und innere Energie. Weiters wird ein Modell für die Wachstumsterme vorgeschlagen, dessen Form sich an das Diffusionsgesetz von STEFAN anlehnt sowie an eine Formulierung von SEELIGER für Planeten mit veränderlicher Masse. Es wird gezeigt, daß einige aktuelle biomechanische Formulierungen aus der Literatur in diesem Modell als Spezialfälle enthalten sind, und es wird ein scheinbarer Widerspruch zwischen diesen Formulierungen aufgeklärt.

\section{Einleitung}

Im vorliegenden Beitrag wird die Eulersche Darstellung der nichtrelativistischen Kontinuumsmechanik verwendet. Im Rahmen dieser Theorie können die lokalen Bilanzgleichungen in der folgenden 
allgemeinen Form geschrieben werden:

$$
\overline{(\rho \Psi)}+\rho \Psi \operatorname{div} v=S[\Psi]-\operatorname{div} I[\Psi] .
$$

Der räumliche Divergenzoperator wird mit „div“ a abgekürzt. Der über einen Ausdruck gesetzte Punkt steht für die materielle Zeitableitung, welche von einem inertialen Beobachter ermittelt wird. Die Massendichte wird mit $\rho$ bezeichnet, und es steht

$$
v=\overline{\left(x-x_{0}\right)}=\dot{r}
$$

für die Absolutgeschwindigkeiten der Teilchen des betrachteten materiellen Körpers. Der Verbindungsvektor des momentanen Ortes $x$ eines Teilchens von einem festen Ort $x_{0}$ heißt $r$. Die Größe $\Psi$ steht für einen Skalar oder Vektor, welcher den Teilchen in mechanisch sinnvoller Weise zugeordnet ist. Verschwindet die rechte Seite von Gl. (1) überall im Körper, so bleibt das Integral von $\rho \Psi$ in einem materiellen Volumen erhalten. Ist dies nicht der Fall, so sorgen die Quellterme $S[\Psi]$ und $I[\Psi]$ auf der rechten Seite von Gl. (1) für eine entsprechende Bilanz. Die unterschiedlichen mechanischen Bedeutungen von $S[\Psi]$ und $I[\Psi]$ erschließen sich aus der integralen Form von Gl. (1) für ein materielles Volumen im Körper. (Die Oberfläche eines materiellen Volumens bewegt sich mit den Teilchen mit, welche sich auf dieser Oberfläche befinden. Auf das Anschreiben der integralen Bilanzgleichungen wird im folgenden aus Platzgründen verzichtet.)

Es steht $S[\Psi]$ (im Englischen supply genannt) für die Dichte von Quellen, die im Inneren des materiellen Volumens verteilt sind; ihr Volumenintegral trägt zu einer zeitlichen Änderung des Integrals von $\rho \Psi$ über das materielle Volumen bei. Es hat $S[\Psi]$ die Dimension einer Rate von $\rho \Psi$. Wählt man z. B. $\Psi=1$, so liefert Gl. (1) die lokale Form der Kontinuitätsgleichung, also des Satzes von der Bilanz der Masse, welche in einem materiellen Volumen eingeschlossen ist. In klassischen Formulierungen bleibt diese mit $S[\Psi=1]=S[1]=0$ und $I[1]=0$ erhalten. Wird die Masse aber nicht erhalten, so steht $S$ [1] für verteilte Massequellen im Inneren, mit der Dimension einer Massendichte pro Zeiteinheit.

Weiters steht der influx $I[\Psi]$ in Gl. (1) für die Dichte von Quellen, die über die Oberfläche des materiellen Volumens verteilt wirken und deren Oberflächenintegral ebenfalls zu einer zeitlichen Änderung des Integrals von $\rho \Psi$ über das materielle Volumen beiträgt. $I[\Psi]$ hat also die Dimension einer Rate von $\rho \Psi$ mal Längeneinheit. Bei der Herleitung von Gl. (1) aus der integralen Bilanzgleichung (mit Hilfe 
des Divergenztheorems) muß angenommen werden, daß sich $I[\Psi]$ in hinreichend stetiger Weise von der Oberfläche ins Innere des materiellen Volumens hinein fortsetzen läßt, so daß dort der räumliche Divergenzoperator „div“ angewendet werden darf. Wählt man z. B. $\Psi=v$, so steht $\rho \Psi$ für den Impuls pro Volumeneinheit, und es liefert Gl. (1) die lokale Form des Impulssatzes, das sogenannte dynamische Grundgesetz. Für den entsprechenden influx gilt dann

$$
I[v]=-\tau,
$$

wo $\tau$ den Cauchyschen Spannungstensor bezeichnet, mit der Dimension einer Kraft pro Fläche. Dieser Tensor ist über das Cauchysche Fundamentaltheorem mit dem Spannungsvektor an der (von der Umgebung freigeschnitten gedachten) Oberfläche des materiellen Volumens verknüpft. Die Resultierende der Oberflächenkräfte, also das Oberflächenintegral über die entsprechenden Spannungsvektoren, trägt zu einer zeitlichen Änderung des Gesamtimpulses im materiellen Volumen bei.

Allgemein gilt: Falls $\Psi$ in Gl. (1) eine skalare Größe ist, so steht $S[\Psi]$ ebenfalls für einen Skalar, und $I[\Psi]$ ist ein Vektor. Steht hingegen $\Psi$ für einen Vektor, dann ist $S[\Psi]$ ebenfalls ein Vektor, $I[\Psi]$ aber steht für einen Tensor (2. Stufe). In der vorliegenden Arbeit wird für die Verknüpfung von Skalaren, Vektoren und Tensoren eine dyadische Notation herangezogen, die auf GIBBS zurückgeht, siehe WILSON [1]. Speziell wird eine Schreibweise in Anlehnung an den Handbuchartikel von SERRIN [2] verwendet. Für eine Diskussion der Stetigkeitsanforderungen, welche zur Gültigkeit von Gl. (1) benötigt werden, wird auf den Handbuchartikel von TRUESDELL und TOUPIN [3] verwiesen. In [3] wird hervorgehoben, daß die rechte Seite von Gl. (1) immer in einem einzigen, effektiven Quellterm zusammengefaßt werden kann, wovon im folgenden Gebrauch gemacht wird. Es sei angemerkt, daß eine solche Zusammenfassung dann zweckmäßig erscheint, wenn die unterschiedlichen mechanischen Bedeutungen von $S[\Psi]$ und $I[\Psi]$ im einzelnen nicht vorgegeben sind. Im folgenden werden wir mit effektiven supply-Termen arbeiten.

In der Kontinuumsmechanik werden neben den schon angesprochenen Bilanzgleichungen für Masse und Impuls noch die Bilanzgleichungen für den Drall und die Energie (der erste Hauptsatz der Thermodynamik) als fundamental angesehen. Die lokalen Formen der fundamentalen Bilanzgleichungen folgen aus entsprechenden integralen Bilanzen für ein materielles Volumen, und sie haben den Aufbau der Gl. (1). Aus der Thermodynamik wird in der Kontinuumsmechanik neben dem ersten Hauptsatz auch der zweite 
Hauptsatz benötigt, welcher eine Ungleichung darstellt, also keine Bilanzgleichung im Sinn von Gl. (1) ist. Der zweite Hauptsatz wird in der vorliegenden Arbeit nicht behandelt.

Als Konsequenz der lokalen Form der Bilanz des Impulses, also des dynamischen Grundgesetzes, können durch skalare oder vektorielle Multiplikation die lokalen Bilanzgleichungen für die kinetische Energie beziehungsweise für das Impulsmoment hergeleitet werden, welche beide ebenfalls von der Form der Gl. (1) sind. Integriert man diese lokalen Beziehungen über ein materielles Volumen, so erhält man den Leistungssatz beziehungsweise eine alternative Darstellung des Drallsatzes. Setzt man die lokale Bilanzgleichung für die kinetische Energie in den ersten Hauptsatz der Thermodynamik ein, so ergibt sich als weitere Konsequenz eine lokale Bilanzgleichung für die innere Energie. Die lokalen Formen der Bilanzgleichungen für die kinetische Energie, das Impulsmoment und die innere Energie, welche im folgenden als abgeleitete Bilanzen bezeichnet werden, finden auf Grund ihrer anschaulichen physikalischen Bedeutung in der Kontinuumsmechanik häufig Anwendung, zumal die Herleitung aus dem dynamischen Grundgesetz auch didaktisch günstig ist, siehe ZIEGLER [4], [5].

Die in den Bilanzgleichungen der klassischen Kontinuumsmechanik auftretenden Einzelterme stehen seit langer Zeit fest. Auch die Untersuchungen über polare Kontinua, deren Teilchen neben der Geschwindigkeit eine unabhängige Winkelgeschwindigkeit zugeordnet wird, und wo in den Quelltermen zusätzlich Momentenspannungen und verteilte äußere Momente zu berücksichtigen sind, fanden bereits einen gewissen Abschluß, siehe z. B. CowIN [6] für eine Diskussion der zugehörigen fundamentalen Bilanzgleichungen. Im folgenden beschränken wir uns auf den nicht-polaren Fall. Die in der vorliegenden Arbeit als klassisch bezeichneten Formulierungen nehmen jedenfalls an, daß jedem Ort im Raum momentan nur ein Teilchen zugeordnet ist und daß die Masse in einem materiellen Volumen erhalten bleibt.

Im Gegensatz zur klassischen Kontinuumsmechanik wird in der Theorie chemisch reaktiver Mischungen angenommen, daß einem bestimmten Ort im Raum momentan mehrere Teilchen zugeordnet sind, für jeden Bestandteil der Mischung eines. Diese Teilchen tauschen zufolge chemischer Reaktionen Masse, aber auch Impuls, Drall und Energie untereinander aus, siehe KELLY [7] und TRUESDELL [8] für grundlegende Darstellungen und vergleiche z. B. MoRLAND und SELLERS [9] für eine aktuelle Untersuchung. Formuliert man die lokalen Bilanzgleichungen der Kontinuumsmechanik für einen 
Einzelbestandteil der Mischung, dann müssen zufolge des genannten Austauschs zusätzlich zu den klassischen Termen sogenannte Wachstumsterme (growth terms) eingeführt werden. Zwar kommt es in manchen Fällen nicht zu einem Austausch von Masse, sondern nur zu einem Austausch von Impuls oder Energie, etwa bei der Theorie flüssigkeitsdurchströmter poröser Körper, siehe DE BOER [10], [11] für umfassende Darstellungen. In letzter Zeit wird die Annahme, daß die Masse in einem materiellen Volumen erhalten bleibt, aber allgemein häufig aufgegeben. Dies gilt besonders für das Studium des Wachstums von biologischen Materialien, siehe EPSTEIN und MAUGIN [12], Lubarda und Hoger [13], Zaixing Huang [14] und z. B. AMBROSI und MoLLICA [15] speziell für das Wachstum eines Tumors. Die Form der entsprechenden biomechanischen Bilanzgleichungen in [12]-[15] erinnert zwar an die Theorie chemisch reaktiver Mischungen, aber es wird nicht direkt von dieser Gebrauch gemacht. Jedenfalls treten in den genannten biomechanischen Formulierungen ebenfalls Wachstumsterme auf. Für eine Übersicht zur Literatur von mechanischen Systemen ohne Massenerhaltung sei auch auf IRSCHIK und HOLL [16], [17] verwiesen, wo weitere Anwendungsgebiete mit nichtklassischen Termen in den Bilanzgleichungen diskutiert werden. Um alle diese nicht-klassischen Fälle zu berücksichtigen, schreiben wir an Stelle von Gl. (1)

$$
\begin{aligned}
\overline{(\rho \Psi)}+\rho \Psi \operatorname{div} v & =S^{0}[\Psi]+S^{+}[\Psi], \\
S^{0}[\Psi] & =s[\Psi]-\operatorname{div} i[\Psi],
\end{aligned}
$$

wobei $s[\Psi]$ den supply, und $i[\Psi]$ den influx aus den klassischen Formulierungen der Kontinuumsmechanik darstellt. Die entsprechenden klassischen Terme $s[\Psi]$ und $i[\Psi]$ finden sich z. B. bei LIU [18] für den in der vorliegenden Arbeit behandelten nicht-polaren Fall übersichtlich zusammengestellt. In Gl. (4.2) werden sie zum späteren Gebrauch zu einem effektiven klassischen supply $S^{0}[\Psi]$ zusammengefaßt. Der zusätzliche supply $S^{+}[\Psi]$ in Gl. (4.1) faßt die Wachstumsterme in den oben genannten nicht-klassischen Theorien effektiv zusammen. Er wird im folgenden als Wachstumsterm, oder kurz als Wachstum, der jeweils zu bilanzierenden Größe bezeichnet und kann, entsprechend den tatsächlich vorliegenden physikalischen Gegebenheiten, wiederum in einen supply- und einen influx-Anteil zerlegt werden, analog zu den rechten Seiten von Gln. (1) und (4.2). Eine solche Unterteilung findet man bei EPSTEIN und MAUGIN [12].

Die vorliegende Arbeit verfolgt zwei Ziele. Zunächst werden Beziehungen hergeleitet, welche notwendigerweise gelten müssen 
zwischen den Wachstumstermen in den fundamentalen Bilanzgleichungen, also für Masse, Impuls, Drall und Energie, und den Wachstumstermen in den abgeleiteten Formen, also für kinetische Energie, Impulsmoment und innere Energie. Dafür werden in Abschnitt 2 die fundamentalen lokalen Bilanzgleichungen bei Anwesenheit von Wachstumstermen formuliert. In Abschnitt 3 werden zusätzliche Beziehungen hergeleitet, und zwar aus dem dynamischen Grundgesetz durch skalare und vektorielle Multiplikation sowie aus dem ersten Hauptsatz der Thermodynamik. Diese abgeleiteten Beziehungen sind ebenfalls von der allgemeinen Form der lokalen Bilanzgleichungen, Gl. (4.1), und werden somit in Abschnitt 4 formal als lokale Bilanzgleichungen für kinetische Energie, Impulsmoment und innere Energie angeschrieben. Ein Vergleich der Gleichungen in Abschnitt 3 und 4 liefert dann die gesuchten Beziehungen zwischen den Wachstumstermen in den fundamentalen und den abgeleiteten Bilanzgleichungen. Es wird weiters gezeigt, daß analoge Beziehungen für die klassischen supply-Terme gelten. Schließlich wird eine Formulierung für die Wachstumsterme in den fundamentalen Bilanzgleichungen vorgeschlagen, welche sich an das Diffusionsgesetz von STEFAN [19] anlehnt, beziehungsweise an eine Formulierung von SEELIGER für Planeten mit veränderlicher Masse [20]. Es wird gezeigt, daß die genannten biomechanischen Formulierungen in [13]-[15] als Spezialfälle in der vorgeschlagenen Formulierung enthalten sind, und es werden die zugehörigen abgeleiteten Wachstumsterme angegeben. Ein scheinbarer Widerspruch zwischen diesen Formulierungen wird aufgeklärt.

\section{Die fundamentalen Bilanzgleichungen in lokaler Form mit Wachstumstermen}

Wir beginnen mit der lokalen Bilanz der Masse, welche aus Gl. (4) mit $\Psi=1$ folgt. In der klassischen Theorie bleibt die Masse erhalten, so daß $s[1]=0$ und $i[1]=0$. Gleichung (4) lautet dann

$$
\begin{aligned}
\dot{\rho}+\rho \operatorname{div} v & =S^{0}[1]+S^{+}[1], \\
S^{0}[1] & =0,
\end{aligned}
$$

wobei $S^{+}[1]$ der Wachstumsterm für die Masse ist; der klassische supply $S^{0}[1]$ verschwindet. Rückeinsetzen der erweiterten Kontinuitätsgleichung (5.1) in Gl. (4) liefert die allgemeine Form der lokalen Bilanzgleichung in Anwesenheit eines Massenwachstums,

$$
\rho \dot{\Psi}+\Psi S^{+}[1]=S^{0}[\Psi]+S^{+}[\Psi] .
$$


Der nächste Schritt ist die lokale Bilanz des Impulses. Wir setzen also $\Psi=v$ als Impuls pro Masseneinheit. Mit den Impulsquellen $S^{0}[v]$ aus der klassischen Form des lokalen Impulssatzes ergibt Gl. (6)

$$
\begin{aligned}
\rho \dot{v}+v S^{+}[1] & =S^{0}[v]+S^{+}[v], \\
S^{0}[v] & =\rho b+\operatorname{div} \tau,
\end{aligned}
$$

wobei $b$ die Körperkraft je Masseneinheit im Inneren ist und $S^{+}[v]$ das zusätzliche Impulswachstum darstellt. Der Cauchysche Spannungstensor $\tau$ kommt wie gesagt bei der Herleitung von Gl. (7) aus der integralen Form der Impulsbilanz ins Spiel, und zwar zufolge der Kräfte an der Oberfläche. Die Körperkräfte im Inneren und die Oberflächenkräfte werden als äußere Kräfte bezeichnet. Gleichung (7.1) stellt das dynamische Grundgesetz in Anwesenheit von Wachstumstermen dar.

Wir setzen mit der lokalen Bilanz des Dralls fort, mit $\Psi=\alpha=$ $r \times v$ als Drall pro Masseneinheit (im nicht-polaren Fall). Dabei steht $r$ für den Ortsvektor des betrachteten Teilchens von einem raumfesten Bezugspunkt, siehe Gl. (2), und das Symbol „× " kennzeichnet das vektorielle Produkt. Es gilt:

$$
\begin{aligned}
\rho \dot{\alpha}+\alpha S^{+}[1] & =S^{0}[\alpha]+S^{+}[\alpha], \\
S^{0}[\alpha] & =r \times \rho b+\operatorname{div}(r \times \tau),
\end{aligned}
$$

wobei $S^{+}[\alpha]$ das zusätzliche Drallwachstum ist und $S^{0}[\alpha]$ für die klassischen Drallquellen steht. Es wird angenommen, daß weder im Inneren des Körpers noch an seiner Oberfläche verteilte äußere Momente eingeprägt sind. Das vektorielle Produkt $r \times \rho b$ steht für einen Vektor, während $r \times \tau$ ein Tensor (2. Stufe) ist. Der Ausdruck $S^{0}[\alpha]$ folgt aus dem integralen Drallsatz, und zwar aus dem resultierenden Moment der äußeren Kräfte um den raumfesten Bezugspunkt.

Schließlich lautet die lokale Form des ersten Hauptsatzes der Thermodynamik mit $\Psi=\Lambda$ als Energie pro Masseneinheit:

$$
\begin{aligned}
\rho \dot{\Lambda}+\Lambda S^{+}[1] & =S^{0}[\Lambda]+S^{+}[\Lambda], \\
S^{0}[\Lambda] & =\rho(b \cdot v+q)+\operatorname{div}(\tau \cdot v+h) .
\end{aligned}
$$

Das zusätzliche Wachstum der Energie wird mit $S^{+}[\Lambda]$ bezeichnet. In Gl. (9.2) steht $\rho q$ für den supply der Energie zufolge nichtmechanischer Energiequellen, und der Vektor $(-h)$ ist der nichtmechanische influx. Die skalare Multiplikation wird durch einen Punkt zwischen den betroffenen Termen angezeigt. Das skalare Produkt $b \cdot v$ ist ein Skalar, während $\tau \cdot v$ für einen Vektor steht. 
Der Term $\rho b \cdot v+\operatorname{div}(\tau \cdot v)$ ist die Leistungsdichte der äußeren Kräfte (unter Berücksichtigung der ins Innere fortgesetzten Oberflächenkräfte).

\section{Abgeleitete Bilanzgleichungen in lokaler Form mit Wachstumstermen}

Nun liegt es nahe, zu einer alternativen Formulierung der lokalen Bilanzgleichung für den Drall dadurch zu kommen, daß man das vektorielle Produkt des Ortsvektors $r$ mit dem dynamischen Grundgesetz, Gl. (7), bildet. In Gl. (8.1) ist ja $\alpha=r \times v$ und damit $\dot{\alpha}=r \times \dot{v}$, weil $\dot{r} \times v=v \times v=0$. Man erhält also aus Gl. (7)

$$
\rho \dot{\alpha}+\alpha S^{+}[1]=r \times \rho b+r \times \operatorname{div} \tau+r \times S^{+}[v] .
$$

Es gilt weiters der Zusammenhang

$$
r \times \operatorname{div} \tau=-\tau_{\times}+\operatorname{div}(r \times \tau),
$$

mit dem Gibbsschen Kreuzvektor $\tau_{\times}$. Dieser ist ein axialer Vektor, welcher dem anti-metrischen Teil des Spannungstensors $\tau$ zugeordnet ist. Gleichung (11) bringt also die Frage ins Spiel, ob der Spannungstensor symmetrisch ist. Einsetzen von Gl. (11) in Gl. (10) liefert

$$
\rho \dot{\alpha}+\alpha S^{+}[1]=r \times \rho b-\tau_{\times}+\operatorname{div}(r \times \tau)+r \times S^{+}[v] .
$$

Diese Beziehung gilt alternativ zu Gl. (8).

Ebenso liegt es nahe, zu einer lokalen Bilanzgleichung für die kinetische Energie zu gelangen, indem man das dynamische Grundgesetz, Gl. (7), skalar mit $v$ multipliziert. Die kinetische Energie pro Masseneinheit ist

$$
\kappa=\frac{1}{2} v \cdot v
$$

mit

$$
\dot{\kappa}=\dot{v} \cdot v .
$$

Berücksichtigt man den Zusammenhang

$$
(\operatorname{div} \tau) \cdot v=-\tau: \operatorname{grad} v+\operatorname{div}(\tau \cdot v),
$$

wobei der Doppelpunkt für das kontrahierte (skalare) Tensorprodukt steht und der räumliche Gradientenoperator mit ,grad“ abgekürzt wird, so erhält man aus Gl. (7)

$$
\rho \dot{\kappa}+2 \kappa S^{+}[1]=\rho b \cdot v-\tau: \operatorname{grad} v+\operatorname{div}(\tau \cdot v)+v \cdot S^{+}[v] .
$$


Der Ausdruck $(-\tau: \operatorname{grad} v)$ steht für die Leistungsdichte der inneren Kräfte.

Nun kann die Energie pro Masseneinheit, $\Lambda$, unterteilt werden in die kinetische Energie pro Masseneinheit, $\kappa$, und in die innere Energie pro Masseneinheit, $\varepsilon$ :

$$
\Lambda=\kappa+\varepsilon \text {. }
$$

Setzt man dies in den ersten Hauptsatz der Thermodynamik, Gl. (9), ein, und zieht man Gl. (16) ab, so folgt

$$
\rho \dot{\varepsilon}+\varepsilon S^{+}[1]=\rho r+\tau: \operatorname{grad} v+\operatorname{div} h+S^{+}[\Lambda]+\kappa S^{+}[1]-v \cdot S^{+}[v] .
$$

Die Beziehungen in den Gln. (12), (16) und (18) dieses Abschnittes bilden eine wichtige Grundlage für die nachfolgenden Untersuchungen.

\section{Notwendige Beziehungen zwischen den fundamentalen und den hergeleiteten Wachstumstermen}

Ein Vergleich zeigt, daß die aus dem dynamischen Grundgesetz hergeleiteten Beziehungen, Gln. (12), (16) und (18), von der allgemeinen Form der lokalen Bilanzgleichung, Gl. (6), sind, weshalb wir im folgenden entsprechende Bilanzgleichungen anschreiben.

Wir beginnen mit Gl. (12) und formulieren diese Beziehung als

$$
\begin{aligned}
\rho \dot{\alpha}+\alpha S^{+}[1] & =S_{1}^{0}[\alpha]+S_{1}^{+}[\alpha], \\
S_{1}^{0}[\alpha] & =r \times \rho b-\tau_{\times}+\operatorname{div}(r \times \tau) .
\end{aligned}
$$

Aus Gründen der Unterscheidung ist es vorteilhaft, Gl. (19.1) als lokale Form des Impulsmomentensatzes zu bezeichnen, um nämlich daran zu erinnern, daß Gl. (12) aus dem vektoriellen Produkt des Ortsvektors mit der lokalen Bilanz für den Impuls resultiert. Der effektive supply $S_{1}^{0}[\alpha]$ in Gl. (19.2) entspricht der klassischen Formulierung des Impulsmomentensatzes, und der Ausdruck $S_{1}^{+}[\alpha]$ ist das Wachstum des Impulsmoments. Ein Vergleich von Gl. (12) mit Gl. (19.1) liefert die folgende notwendige Beziehung zwischen Impulsmomentenwachstum und Impulswachstum:

$$
S_{1}^{+}[\alpha]=r \times S^{+}[v] .
$$

Speziell muß das Wachstum des Impulsmoments verschwinden, wenn das Impulswachstum $S^{+}[v]$ verschwindet.

Vergleicht man andererseits den Drallsatz, Gl. (8), mit Gln. (19) und (20), so erhält man eine Beziehung zwischen dem Gibbsschen 
Kreuzvektor des Cauchyschen Spannungstensors, dem Drallwachstum und dem Impulswachstum:

$$
\tau_{\times}=S^{+}[\alpha]-r \times S^{+}[v] .
$$

Der Cauchysche Spannungstensor $\tau$ ist also nur dann symmetrisch, wenn die rechte Seite von Gl. (21) verschwindet.

Einsetzen von Gl. (20) in Gl. (21) ergibt weiters die folgende notwendige Beziehung zwischen dem Impulsmomentenwachstum und dem Drallwachstum

$$
S_{1}^{+}[\alpha]=S^{+}[\alpha]-\tau_{\times},
$$

welche alternativ zu Gl. (20) verwendet werden kann.

Die in Gl. (16) angegebene Beziehung ist ebenfalls von der Form der Gl. (6). Wir schreiben also formal

$$
\begin{aligned}
\rho \dot{\kappa}+\kappa S^{+}[1] & =S^{0}[\kappa]+S^{+}[\kappa], \\
S^{0}[\kappa] & =\rho b \cdot v-\tau: \operatorname{grad} v+\operatorname{div}(\tau \cdot v) .
\end{aligned}
$$

Die Beziehung in Gl. (23.1) wird die lokale Bilanzgleichung für die kinetische Energie genannt. Das Wachstum der kinetischen Energie wird mit $S^{+}[\kappa]$ bezeichnet, und $S^{0}[\kappa]$ ist der effektive supply der klassischen Formulierung der Bilanz der kinetischen Energie. Integriert man über das betrachtete Körpervolumen auf, so erhält man den Leistungssatz, siehe ZIEGLER [4] für den klassischen Fall. Der Vergleich von Gl. (23) und Gl. (16) ergibt die folgende Beziehung zwischen dem Wachstum der kinetischen Energie, dem Wachstum der Masse und dem Impulswachstum:

$$
S^{+}[\kappa]=-\kappa S^{+}[1]+v \cdot S^{+}[v] .
$$

Das Wachstum der kinetischen Energie verschwindet somit, wenn die rechte Seite von Gl. (24) verschwindet.

Auch die in Gl. (18) festgestellte Beziehung hat die Form der allgemeinen lokalen Bilanzgleichung, Gl. (6). Wir schreiben deshalb formal

$$
\begin{aligned}
\rho \dot{\varepsilon}+\varepsilon S^{+}[1] & =S^{0}[\varepsilon]+S^{+}[\varepsilon], \\
S^{0}[\varepsilon] & =\rho r+\tau: \operatorname{grad} v+\operatorname{div} h .
\end{aligned}
$$

Dies ist die erweiterte lokale Form der Bilanz der inneren Energie, mit dem Wachstum der inneren Energie $S^{+}[\varepsilon]$ und mit dem klassischen supply $S^{0}[\varepsilon]$. Der Vergleich von Gl. (25) mit Gl. (18) liefert nach Einsetzen von Gl. (24) die folgende Beziehung zwischen dem 
Wachstum der inneren Energie und den Wachstumstermen für Masse, Impuls und Energie:

$$
S^{+}[\varepsilon]=\kappa S^{+}[1]-v \cdot S^{+}[v]+S^{+}[\Lambda] .
$$

Dies ist gleichbedeutend mit

$$
S^{+}[\kappa]+S^{+}[\varepsilon]=S^{+}[\Lambda] .
$$

Also muß die Summe aus den Wachstumstermen für innere und kinetische Energie gleich sein dem Energiewachstum, was zu erwarten war.

In den Gln. (20), (24) und (26) wurden Beziehungen angegeben, welche notwendigerweise gelten müssen zwischen den Wachstumstermen für Impulsmoment, kinetische Energie bzw. innere Energie, also den Wachstumstermen in den hergeleiteten Bilanzgleichungen, und den fundamentalen Wachstumstermen für Masse, Impuls, Drall und Energie. Es läßt sich nun zeigen, daß die klassischen supplyTerme in den abgeleiteten Bilanzgleichungen in genau der gleichen Weise von den klassischen supply-Termen in den fundamentalen Bilanzgleichungen abhängen, wie dies für die Wachstumsterme in den Gln. (20), (24) und (26) der Fall ist, also

$$
\begin{aligned}
S_{1}^{0}[\alpha] & =r \times S^{0}[v], \\
S^{0}[\kappa] & =-\kappa S^{0}[1]+v \cdot S^{0}[v], \\
S^{0}[\varepsilon] & =\kappa S^{0}[1]-v \cdot S^{0}[v]+S^{0}[\Lambda] .
\end{aligned}
$$

Dies kann leicht durch Einsetzen der Definitionen in Gln. (5.2), (7.2), (8.2) und (9.2) einerseits, sowie jener in Gln. (19.2), (23.2) und (25.2) andererseits, und unter Verwendung der Gln. (11) und (15) überprüft werden. Die Beziehungen in Gln. (28)-(30) erscheinen für sich interessant, und sie belegen die Zweckmäßigkeit der vorgelegten Methodik, bei welcher die klassischen supply-Terme und die Wachstumsterme auf der rechten Seite von Gl. (6.1) gleichartig behandelt werden.

\section{Ein Wachstumsmodell}

Für das Wachstum wird folgendes Modell vorgeschlagen: Es soll jedem der betrachteten Teilchen Masse mit der Massendichterate $S^{+}[1]$ zuwachsen. Dieser Massezuwachs soll zufolge fiktiver Teilchen erfolgen, die an den betrachteten Teilchen haften bleiben. Es sei $v_{f}$ die Geschwindigkeit und $\varepsilon_{f}$ die innere Energie pro Masseneinheit 
dieser zuwachsenden fiktiven Teilchen vor dem Haftenbleiben. Auf Grund des genannten Modells kann man setzen

$$
\begin{aligned}
S^{+}[v] & =v_{f} S^{+}[1], \\
S^{+}[\alpha] & =r \times v_{f} S^{+}[1], \\
S^{+}[\Lambda] & =\left(\kappa_{f}+\varepsilon_{f}\right) S^{+}[1],
\end{aligned}
$$

mit der fiktiven kinetischen Energie pro Masseneinheit

$$
\kappa_{f}=\frac{1}{2} v_{f} \cdot v_{f} \text {. }
$$

Speziell erhält man dann für das dynamische Grundgesetz, Gl. (7.1), sowie für den ersten Hauptsatz der Thermodynamik, Gl. (9.1),

$$
\begin{aligned}
\rho \dot{v} & =S^{0}[v]+\left(v_{f}-v\right) S^{+}[1], \\
\rho \dot{\Lambda} & =S^{0}[\Lambda]+\left(\kappa_{f}+\varepsilon_{f}-\Lambda\right) S^{+}[1],
\end{aligned}
$$

mit der Energie $\Lambda$ aus Gl. (17).

Das in Gl. (33.1) vorgeschlagene dynamische Grundgesetz ist von der Art der bekannten Raketengleichung. Eine solche Beziehung wurde für punktförmige Massen zuerst von SEELIGER [20] am Beispiel von Planeten hergeleitet, welche kontinuierlich Masse mit der Geschwindigkeit $v_{f}$ gewinnen oder verlieren, siehe [16] für eine ausführlichere Diskussion. Man kann auch argumentieren, daß der Term $\left(v_{f}-v\right) S^{+}[1]$ in Gl. (33.1) in Analogie zum Impulswachstum zufolge von Teilchen steht, welche mit der Geschwindigkeit $v_{f}$ in den Bestandteil einer Mischung hinein diffundieren. Die Gl. (33.1) entsprechende Diffusionstheorie geht auf STEFAN [19] zurück, siehe ebenfalls [16]. In der Diffusionstheorie hat $S^{+}[1]$ allerdings nicht die Bedeutung eines Massenwachstums, sondern eines Diffusionskoeffizienten der Mischung aus zwei Bestandteilen ohne Massenwachstum. Die biomechanischen Wachstumsformulierungen in den Arbeiten [12]-[15] beziehen sich nicht direkt auf die Theorie der Mischungen, und das Massenwachstum steht bei ihnen im Vordergrund. Im vorliegenden Zusammenhang wird deshalb die einführend zu Gl. (31) genannte Interpretation gegenüber der Diffusionstheorie bevorzugt. Einsetzen von Gl. (31) in die Gln. (20), (24) und (26) liefert die folgenden Ausdrücke für die abgeleiteten Wachstumsterme:

$$
\begin{aligned}
S_{1}^{+}[\alpha] & =r \times v_{f} S^{+}[1], \\
S^{+}[\kappa] & =v \cdot\left(-\frac{1}{2} v+v_{f}\right) S^{+}[1], \\
S^{+}[\varepsilon] & =\left(\frac{1}{2}\left(v-v_{f}\right) \cdot\left(v-v_{f}\right)+\varepsilon_{f}\right) S^{+}[1] .
\end{aligned}
$$


Somit verschwindet mit Gl. (21) auch der Gibbssche Kreuzvektor

$$
\tau_{\times}=0,
$$

so daß der Cauchysche Spannungstensor im Rahmen des vorliegenden Modells symmetrisch wird.

Wir wenden uns nun den Formulierungen in der genannten biomechanischen Literatur zu. Setzt man in Gl. (34)

$$
v_{f}=v \text {, }
$$

nimmt man also an, daß die fiktiven Teilchen die gleiche Geschwindigkeit haben wie die betrachteten Teilchen, so folgt für das dynamische Grundgesetz, Gl. (33.1), und den ersten Hauptsatz, Gl. (33.2),

$$
\begin{aligned}
\rho \dot{v} & =S^{0}[v], \\
\rho \dot{\Lambda} & =S^{0}[\Lambda]+\left(\varepsilon_{f}-\varepsilon\right) S^{+}[1],
\end{aligned}
$$

weil $\kappa_{f}=\kappa$. Die Gl. (37.1) findet man bei AMBrosi und MolliCA [15], Seite 1303, und bei LuBARDA und Hoger [13], Seite 4632. Gleichung (37.2) entspricht einer Beziehung bei LUBARDA und HogER [13], Seite 4634. Die Gln. (34) liefern die folgende Form der abgeleiteten Wachstumsterme

$$
\begin{aligned}
S_{1}^{+}[\alpha] & =r \times v S^{+}[1], \\
S^{+}[\kappa] & =\kappa S^{+}[1], \\
S^{+}[\varepsilon] & =\varepsilon_{f} S^{+}[1] .
\end{aligned}
$$

Alternativ zu Gl. (36) nehmen wir noch an, daß

$$
v_{f}=\frac{1}{2} v \text {. }
$$

Dann liefert Gl. (33.1) die folgende Form des dynamischen Grundgesetzes:

$$
\rho \dot{v}=S^{0}[v]-\frac{1}{2} v S^{+}[1] .
$$

Diese Beziehung wurde von ZaIXING HuAng [14], Seite 957, im Rahmen einer andersartigen Systematik angegeben. Der erste Hauptsatz der Thermodynamik lautet jetzt

$$
\rho \dot{\Lambda}=S^{0}[\Lambda]+\left(\varepsilon_{f}-\frac{3}{4} \kappa-\varepsilon\right) S^{+}[1],
$$


weil $\kappa=4 \kappa_{f}$. Einsetzen von Gl. (39) in Gl. (34) liefert die folgende Form der abgeleiteten Wachstumsterme,

$$
\begin{aligned}
S_{1}^{+}[\alpha] & =\frac{1}{2} r \times v S^{+}[1], \\
S^{+}[\kappa] & =0, \\
S^{+}[\varepsilon] & =\left(\frac{1}{4} \kappa+\varepsilon_{f}\right) S^{+}[1]=\left(\kappa_{f}+\varepsilon_{f}\right) S^{+}[1]=S^{+}[\Lambda] ;
\end{aligned}
$$

siehe Gln. (27) und (31.3). Die Bilanzgleichung für die innere Energie, Gl. (25.1), folgt somit zu

$$
\rho \dot{\varepsilon}+\varepsilon S^{+}[1]=S^{0}[\varepsilon]+S^{+}[\Lambda] .
$$

Diese Beziehung findet sich ebenfalls bei ZAIXING HUANG [14] auf Seite 957, wo allerdings auf das obige Modell des Zuwachsens von fiktiven Teilchen mit eigener Geschwindigkeit und innerer Energie nicht Bezug genommen wird. In [14] wird die spezielle Form des dynamischen Grundgesetzes in Gl. (40.1) wie folgt gefunden. Es wird der erste Hauptsatz der Thermodynamik in der Form der Gl. (18) angeschrieben. Dann wird gefordert, daß diese Beziehung invariant gegenüber einer überlagerten Starrkörperbewegung sein soll. Daraus wird geschlossen, daß die letzten beiden Terme in Gl. (18) verschwinden sollten, weil sie Geschwindigkeitsterme beinhalten, die nicht invariant sind. Das ergibt dann:

$$
S^{+}[v]=\frac{1}{2} v S^{+}[1] .
$$

Einsetzen in das dynamische Grundgesetz, Gl. (7.1), liefert Gl. (40.1). Auf Grund dieser Argumentation erscheint es zunächst, als wäre die Formulierung in [14] den Formulierungen bei AMBROSI und MOLLICA [15] und LuBARDA und HOGER [13] vorzuziehen. Dieser scheinbare Widerspruch kann im Rahmen der vorliegenden Modellbildung aufgeklärt werden.

Zunächst ersetzen wir die Forderung der Invarianz gegenüber einer Starrkörperbewegung durch die Forderung nach der Invarianz gegenüber einem Beobachterwechsel. Diese beiden Forderungen sind im klassischen Fall ohne Massenwachstum äquivalent. Im Rahmen der vorliegenden Modellierung geht es um zwei Klassen von Teilchen, nämlich die betrachteten Teilchen und die zuwachsenden, fiktiven Teilchen, und beide haben unterschiedliche Geschwindigkeiten. In Gl. (18) könnten nur die letzten drei Terme auf der rechten Seite nicht invariant gegenüber einem Beobachterwechsel sein. Diese bilden 
aber gerade das Wachstum der inneren Energie, siehe Gl. (26). (Die Leistungsdichte der inneren Kräfte, $(-\tau: \operatorname{grad} v)$, ist invariant, solang der Cauchysche Spannungstensor symmetrisch ist, siehe LIU [18], und dies gilt im vorliegenden Fall mit Gl. (35).) Wir fordern Invarianz in Gl. (18) also unter Einbeziehung des Wachstums der Energie $S^{+}[\Lambda]$, weil diese auf Grund von Gl. (27) auch das Wachstum der kinetischen Energie beinhaltet und letztere nicht invariant ist gegenüber einem Beobachterwechsel. Ein Vergleich mit Gl. (30) zeigt, daß die letzten drei Terme in Gl. (18) aber gerade unser Wachstum der inneren Energie, $S^{+}[\varepsilon]$, darstellen. Dieses Wachstum soll also invariant gegenüber einem Beobachterwechsel sein. Das ist bei der vorliegenden Modellbildung aber jedenfalls gewährleistet, weil das Wachstum der inneren Energie $S^{+}[\varepsilon]$ in Gl. (34.3) über Geschwindigkeitsdifferenzen definiert ist, welche die geforderte Invarianzeigenschaft gegenüber einem Beobachterwechsel besitzen. Dies gilt somit für alle drei im vorliegenden Abschnitt angesprochenen biomechanischen Formulierungen aus der Literatur, welche also im Hinblick auf Invarianzforderungen gleichberechtigt sind.

\section{Zusammenfassung}

Im vorliegenden Beitrag wurden in Abschnitt 2 die lokalen Bilanzgleichungen für Masse, Impuls (dynamisches Grundgesetz), Drall und Energie (erster Hauptsatz der Thermodynamik) unter Berücksichtigung von entsprechenden Wachstumstermen $S^{+}[1], S^{+}[v]$, $S^{+}[\alpha]$ und $S^{+}[\Lambda]$ in Eulerscher Darstellung formuliert, siehe Gln. (5), (7), (8) und (9). Diese Beziehungen wurden als fundamentale Bilanzgleichungen aufgefaßt. Diese Auffassung steht im Einklang mit der Literatur, siehe z. B. [8]. Aus der lokalen Impulsbilanz, dem dynamischen Grundgesetz, Gl. (7), wurden weiters in Abschnitt 3 Beziehungen hergeleitet, welche dann in Abschnitt 4 als die lokalen Bilanzgleichungen für Impulsmoment und kinetische Energie mit den entsprechenden Wachstumstermen $S_{1}^{+}[\alpha]$ und $S^{+}[\kappa]$ geschrieben wurden, siehe Gln. (19) bzw. (23). Aus dem 1. Hauptsatz der Thermodynamik und der Bilanz der kinetischen Energie ergab sich weiters die Bilanz der inneren Energie mit dem entsprechenden Wachstum $S^{+}[\varepsilon]$, Gl. (25). Aus einem Vergleich der Beziehungen in Abschnitt 3 und 4 wurde dann in Gl. (20) eine Beziehung zwischen Impulsmomentenwachstum $S_{1}^{+}[\alpha]$ und Impulswachstum $S^{+}[v]$ gefunden. Alternativ lieferte Gl. (22) eine Beziehung zwischen Impulsmomentenwachstum und Drallwachstum $S^{+}[\alpha]$, welche den anti-metrischen Teil des Cauchyschen Spannungstensors ins Spiel 
bringt. Dieser verschwindet bei Vorhandensein von Impulswachstum und Drallwachstum im allgemeinen nicht, siehe Gl. (21). Weiters wurde mit Gl. (24) eine Beziehung zwischen dem Wachstum der kinetischen Energie $S^{+}[\kappa]$ und den Wachstumstermen für Masse und Impuls, $S^{+}[1]$ und $S^{+}[v]$, angegeben. Mit Gl. (26) konnte eine Beziehung zwischen dem Wachstum der inneren Energie $S^{+}[\varepsilon]$ und den Wachstumstermen für Masse, Impuls und Energie, $S^{+}[1], S^{+}[v]$ und $S^{+}[\Lambda]$, gewonnen werden. Es wurde weiters festgestellt, daß in den klassischen Formulierungen ohne Wachstum die Quellterme in den abgeleiteten Bilanzgleichungen von jenen in den fundamentalen Bilanzgleichungen in gleicher Weise abhängen, wie dies für die entsprechenden Wachstumsterme gezeigt wurde, siehe Gln. (28)-(30). Mit dieser Analogie sollten die vorgelegten Ergebnisse nicht nur in der Mechanik von Körpern mit Wachstum, sondern auch in der klassischen Kontinuumsmechanik von grundsätzlichem Interesse sein. Im abschließenden Abschnitt 6 wurde mit Gln. (31)-(35) ein Wachstumsmodell vorgestellt, welches verschiedene biomechanische Formulierungen aus der letzten Zeit als Spezialfälle enthält, wobei ein scheinbarer Widerspruch zwischen diesen Theorien beseitigt werden konnte. In der vorliegenden Arbeit wurden Beziehungen zwischen Wachstumstermen im Inneren eines materiellen Volumens untersucht, wobei die betrachteten Felder hinreichend stetig sein müssen. Es sei abschließend angemerkt, daß Beziehungen zwischen Wachstumstermen an singulären Flächen, bei denen also die Felder unterschiedliche Werte an den beiden Seiten der Flächen annehmen, und wo das Wachstum in der Fläche stattfindet, vom Verfasser in [21] hergeleitet wurden.

\section{Literatur}

[1] WiLson, E. B. (1948) Vector Analysis. Yale Univ. Press, New Haven

[2] SERrin, J. (1959) Mathematical principles of classical fluid mechanics. In: FLÜGGE, S. (Hrsg.) Handbuch der Physik, Band VIII/1: Strömungsmechanik I, pp. 125-263. Springer, Berlin

[3] Truesdell, C., Toupin, R. (1960) The classical field theories. In: FlÜGge, S. (Hrsg.) Handbuch der Physik, Band III/1: Prinzipien der Klassischen Mechanik und Feldtheorie, pp. 226-793. Springer, Berlin

[4] ZIEGLER, F. (1998) Mechanics of Solids and Fluids, 2nd english edition, corrected 2nd printing. Springer, New York; first published as ZIEGLER, F. (1985) Technische Mechanik der festen und flüssigen Körper. Springer, Wien-New York

[5] ZIEGLER, F. (1998) Didaktische Aspekte in mechanischen Erhaltungssätzen. GAMM-Mitteilungen 1: 61-72

[6] Cowin, S. C. (1974) The theory of polar fluids. In: Advances in Applied Mechanics (YIH, C.-S., ed.) 14: 279-348 
[7] Kelly, P. D. (1964) A reacting continuum. Int. J. Engineering Sciences 2: $129-153$

[8] Truesdell, C. (1984) Rational Thermodynamics, 2nd Ed. Springer, New York

[9] Morland, L. W., SEllers, S. (2001) Multiphase mixtures and singular surfaces. Int. J. Non-Linear Mechanics 36: 131-146

[10] DE BoER, R. (2000) Theory of Porous Media: Highlights in the Historical Development and Current State. Springer, Berlin

[11] DE BoER, R. (2000) Contemporary progress in porous media theory. Transactions of the ASME, Appl. Mech. Rev. 53: 323-369

[12] Epstein, M., Maugin, G. A. (2000) Thermomechanics of volumetric growth in uniform bodies. Int. J. Plasticity 16: 951-978

[13] Lubarda, V. A., Hoger, A. (2002) On the mechanics of solids with a growing mass. Int. J. Solids Structures 39: 4627-4664

[14] HuAng, Z.-X. (2004) The equilibrium equations and constitutive equations of the growing deformable body in the framework of continuum theory. Int. J. NonLinear Mechanics 39: 951-962

[15] Ambrosi, D., MollicA, F. (2002) On the mechanics of a growing tumor. Int. J. Eng. Sci. 40: 1297-1316

[16] IRSCHIK, H., Holl, H. J. (2004) Mechanics of variable-mass systems - Part 1: Balance of mass and linear momentum. Transactions of the ASME, Appl. Mech. Rev. 57: 145-160

[17] IRSCHIK, H., Holl, H. J. (in preparation) Mechanics of variable-mass systems Part 2: Balance of angular momentum and energy

[18] LIU, I.-S. (2002) Continuum Mechanics. Springer, Berlin

[19] STEFAn, J. (1871) Über das Gleichgewicht und die Bewegung, insbesondere die Diffusion von Gasmengen. Sitzungsber. Akad. Wiss. Wien 63.2: 63-124

[20] SeEliger, H. (1890) Über Zusammenstösse und Theilungen planetarischer Massen. Abh. Königl. Bayer. Akad. Wiss., Cl. II, Abth. II XVII: 459-490

[21] IRSCHIK, H. (2003) On the necessity of surface growth terms for the consistency of jump relations at a singular surface. Acta Mech. 162: 195-211

Anschrift des Verfassers: O. Univ.-Prof. Dipl.-Ing. Dr. Hans Irschik, Institut für Technische Mechanik, Johannes Kepler Universität Linz, A-4040 Linz-Auhof, Österreich. E-Mail: irschik@mechatronik.uni-linz.ac.at. 